\title{
Spin transfer and spin-orbit torques in in-plane magnetized (Ga,Mn)As tracks
}

\author{
L. Thevenard,,${ }^{1, *}$ B. Boutigny, ${ }^{1}$ N. Güsken, ${ }^{1}$ L. Becerra, ${ }^{1}$ C. Ulysse,${ }^{2}$ S. Shihab,${ }^{1}$ A. Lemaître, ${ }^{2}$ \\ J.-V. Kim, ${ }^{2}$ V. Jeudy, ${ }^{3}$ and C. Gourdon ${ }^{1}$ \\ ${ }^{1}$ Sorbonne Universités, UPMC Univ Paris 06, CNRS, Institut des Nanosciences de Paris, 4 place Jussieu, 75252 Paris, France \\ ${ }^{2}$ Centre de Nanosciences et de Nanotechnologies, CNRS, Univ. Paris-Sud, Université Paris-Saclay, 91460 Marcoussis, France \\ ${ }^{3}$ Laboratoire de Physique des Solides, CNRS - Université Paris-Sud, bât. 510, 91405 Orsay, France \\ (Received 15 October 2016; revised manuscript received 10 January 2017; published 16 February 2017)
}

\begin{abstract}
Current-driven domain wall motion is investigated experimentally in in-plane magnetized (Ga,Mn)As tracks. The wall dynamics is found to differ in two important ways with respect to perpendicularly magnetized $(\mathrm{Ga}, \mathrm{Mn})$ As: the wall mobilities are up to ten times higher and the walls move in the same direction as the hole current. We demonstrate that these observations cannot be explained by spin-orbit field torques (Rashba and Dresselhaus types) but are consistent with nonadiabatic spin transfer torque enhanced by the strong spin-orbit coupling of $(\mathrm{Ga}, \mathrm{Mn})$ As. This mechanism opens the way to domain wall motion driven by bulk rather than interfacial spin-orbit coupling as in ultrathin ferromagnet/heavy metal multilayers.
\end{abstract}

DOI: 10.1103/PhysRevB.95.054422

\section{INTRODUCTION}

Nonvolatile memory and logic devices based on magnetic domain-wall (DW) manipulation [1,2] remain technologically challenging as they require narrow DWs, large velocities, and low voltage/current density operation, features that are difficult to combine in a given material. This has led to constant endeavors to optimize the torques experienced by DWs under an applied current. Initial works focused on spin transfer torques (STT [3-7]). Recently, torques originating from the spin-orbit interaction (SOI) have been evidenced in ferromagnetic/nonmagnetic multilayers [8-14]. The mechanisms involved in DW propagation are still partially under debate, but signatures of the Rashba effective field, the spin Hall effect, and the chiral Dzyaloshinskii-Moriya interaction (DMI) have been suggested $[8,11,15,16]$. These observations have been limited to out-of-plane magnetized heavy metal/metallic ferromagnet combinations (Pt/Co/AlOx, GdOx or Gd [13,17], Pt/Co/Ni/Co [12], Pt/ or $\mathrm{Ta} / \mathrm{CoFe} / \mathrm{MgO}[11,14]$ ), in which the main source of SOI was interfacial inversion asymmetry along the sample normal $z$. The purpose of this work is to investigate experimentally the case of a ferromagnet that is its own source of (bulk) spin-orbit interaction, without relying on adjacent layers. For this, we studied the dilute magnetic semiconductor (Ga,Mn)As, in which the complex anisotropy and rich spin-orbit coupling physics enable numerous configurations to be tested.

In $(\mathrm{Ga}, \mathrm{Mn}) \mathrm{As}$, spin-orbit coupling gives rise to two effects very different in magnitude. The main one is described by the Kohn-Luttinger (KL) Hamiltonian. It splits the manifold of valence states with $L=1$ orbital quantum number and $S=$ $1 / 2$ spin into $J=3 / 2$ and $J=1 / 2$ states with $J=L+S$ [18]. For nonzero $k$ wave vectors, the $J=3 / 2$ states are further split into heavy hole $(J= \pm 3 / 2)$ and light hole $(J= \pm 1 / 2)$ states, each with twofold degeneracy. The second and much weaker effect is a lifting of this degeneracy analogous to a $k$-dependent magnetic field. This small spin-orbit effect arises from the lack of centrosymmetry of the zinc-blende lattice

*thevenard@insp.jussieu.fr
( $k^{3}$ Dresselhaus term). A further lowering of the symmetry induced by epitaxial strain $(\varepsilon)$ yields a Dresselhaus term linear in $k$. An even weaker Rashba term, also linear in $k$, exists due to the nonequivalence of [110] and [1 10$]$ directions induced during the growth [19], formally equivalent to an in-plane shear strain or an electric field perpendicular to the interface [18]. This is reminiscent of the one encountered in the $z$-asymmetric metallic stacks mentioned earlier.

These spin-orbit effects have two consequences. First, the heavy-light hole splitting modifies the usual spin transfer torques in the presence of a domain wall since valence states are not pure spin states. A significant hole reflection should occur at the domain wall, resulting in spin accumulation [20-22]. The resulting spin transfer torque is expected to be up to tenfold more efficient than the standard torque but has up to now not been evidenced experimentally. Secondly, Rashba or Dresselhaus spin-orbit splittings of the valence bands result, under current, in an out-of-equilibrium hole spin polarization. The antiferromagnetic exchange interaction between the carrier spins and the Mn spins then yields corresponding effective spin-orbit fields on the magnetization [23-26]. These fields have been evidenced experimentally [27-30] to be in-plane and perpendicular to the current density, but their effect on DWP remains to be shown. Note that these terms are about 100 times weaker than the Rashba term in metals which were claimed to be responsible for fast DWP against the electron direction $[9,13]$.

To explore these spin-orbit interaction effects, we have worked on in-plane magnetized $(\mathrm{Ga}, \mathrm{Mn})$ As tracks, a configuration that has been rarely studied up to now [31,32]. We show that DWs propagate at high mobilities under current and opposite to the direction given by the spin-transfer torque observed in out-of-plane magnetized (Ga,Mn)As [33-37], a radically different phenomenology. We demonstrate our observations to be only partially reconcilable with SO field torques. We suggest instead that they may be a signature of the Kohn-Luttinger Hamiltonian-induced spin accumulation at the domain wall.

\section{SAMPLES AND EXPERIMENTAL SETUP}

DW propagation was studied on a $50 \mathrm{~nm}$ thick epilayer of (Ga,Mn)As grown on (001) GaAs. After an $8 \mathrm{~h} / 200^{\circ} \mathrm{C}$ 
(a) $C_{/ /}$configuration:

(b) $\mathrm{C}_{\perp}$ configuration :

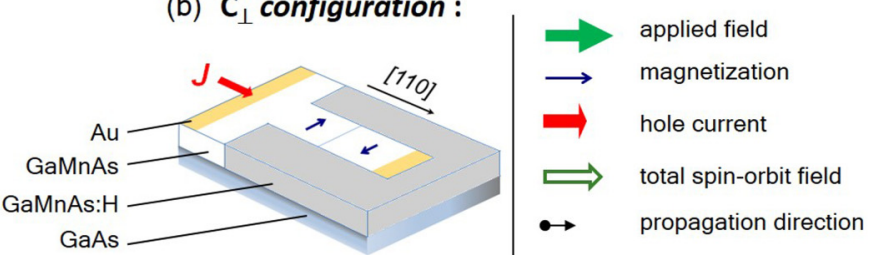

$C_{/ /}$configuration :
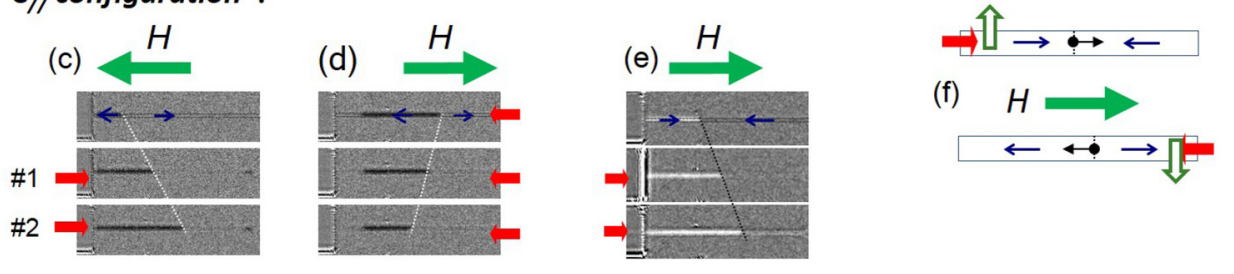

$C_{\perp}$ configuration :

(h) $10 \mu \mathrm{m}, H=0$

(i) $2 \mu \mathrm{m}$

(j)
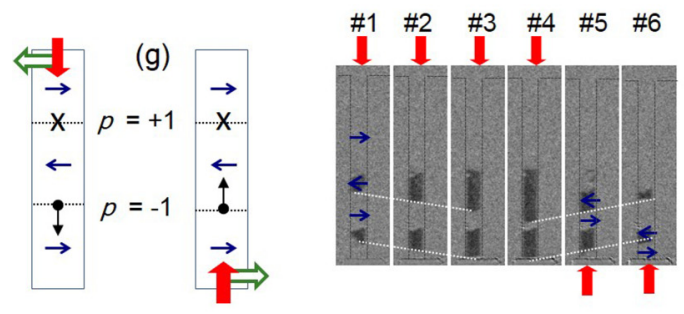

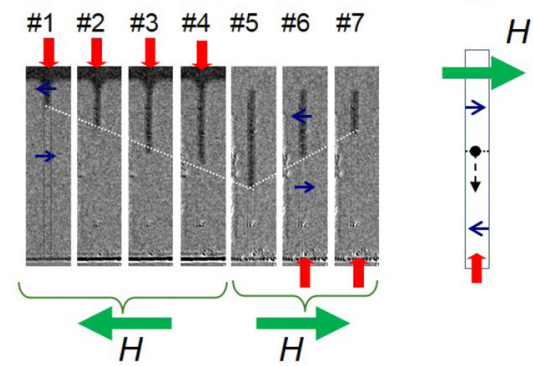

FIG. 1. (a),(b) Two track configurations and device schematics: the hole current (red arrows) flows either parallel ( $\left.\mathrm{C}_{/ /}\right)$or perpendicular $\left(\mathrm{C}_{\perp}\right)$ to the magnetization (dark arrows) whose easy direction is along the crystallographic axis [110]. With the reservoir grounded, the hole current direction shown in this schematics therefore results from a negative potential applied to the opposite tip of the track. The hydrogenated (Ga,Mn)As:H has turned paramagnetic, thus defining the stripe. (c),(g),(j) Phenomenology of current-induced DW motion. The effective spin-orbit field $\left(\vec{H}_{S O}\right)$ is the sum of the Rashba and the Dresselhaus contributions (see text). (d)-(f) and (h),(i) Longitudinal Kerr microscopy images (divided by a reference image taken after saturation) showing domain wall displacements under the application of successive current pulses $(1,2$, etc.). The white dotted lines are guides for the eyes and the black dashed ones materialize the edges of the track and reservoir. (d)-(f) $2 \mu \mathrm{m}$ wide $\mathrm{C}_{/ /}$track, $70 \mathrm{~ns}$ long current pulses of $J=24.5 \mathrm{GA} \mathrm{m}^{-2}$ under $\mu_{0} H=1.1 \mathrm{mT}\left(T_{\text {eff }}=40 \mathrm{~K}\right)$. (h) $10 \mu \mathrm{m} \mathrm{wide} \mathrm{C}_{\perp}$ track, $120 \mathrm{~ns}$ long current pulses of $J=14.9 \mathrm{GA} \mathrm{m}^{-2}$, no applied field $\left(T_{\text {eff }}=80 \mathrm{~K}\right)$. A domain is easily nucleated in the middle of the track under current by $\vec{H}_{S O}$ (pulses 1 and 5). (i) $2 \mu \mathrm{m}$ wide $\mathrm{C}_{\perp}$ track, $100 \mathrm{~ns}$ long current pulses of $J=23.5 \mathrm{GA} \mathrm{m}^{-2}$ under $\mu_{0} H=1.3 \mathrm{mT}\left(T_{\text {eff }}=50 \mathrm{~K}\right.$ ).

anneal, the Curie temperature reached $\mathrm{T}_{C}=116 \mathrm{~K}$ and the magnetically active $\mathrm{Mn}$ concentration $x=3.7 \%$. The layer was grown under compressive strain, which led to the magnetization lying in the plane. SQUID magnetometry and cavity ferromagnetic resonance (FMR) evidenced a uniaxial magnetic anisotropy with the easy axis along the [1 $\overline{10}]$ direction.

The tracks were 2,4 , and $10 \mu \mathrm{m}$ wide and $95 \mu \mathrm{m}$ long, oriented either parallel to the easy axis- " $\mathrm{C}_{/ /}$" configurationor perpendicular to it- " $\mathrm{C}_{\perp}$ " configuration, with one end leading into a large grounded reservoir [Figs. 1(a) and 1(b)]. The reservoir and tracks were patterned by locally passivating the layer at $130{ }^{\circ} \mathrm{C}$ using a hydrogen plasma over a $\mathrm{Ti}$ mask [38]. This selectively turns the (Ga,Mn)As paramagnetic, whilst maintaining the bidimensionality of the layer, thus limiting diffraction-related imaging issues. It also prevents the lattice from relaxing perpendicular to the track, which would pull the easy axis towards the track direction [32], and therefore render $\mathrm{C}_{/ /}$and $\mathrm{C}_{\perp}$ configurations nonequivalent anisotropywise. Finally, $\mathrm{Cr}(10 \mathrm{~nm}) / \mathrm{Au}(200 \mathrm{~nm})$ contacts were thermally evaporated on the sample. Domains were observed with an in-house built longitudinal Kerr microscope, using a $\lambda=635 \mathrm{~nm}$ LED source [39]. A generator was used to apply a continuous or pulsed voltage.

In (Ga,Mn)As, Rashba and Dresselhaus spin-orbit fields are positively (negatively) collinear for $J / /[110](J / /[1 \overline{1} 0] / / x)$, with the total SO effective field $\vec{H}_{S O}$ lying transverse to the tracks [Figs. 1(c) and $1(\mathrm{~g})]$. The field intensity for $J / /[110]$ is about three times larger than for $J / /[1 \overline{1} 0]$ [27-30]. This geometry is distinct from those explored in metallic structures in two respects: (i) the spin-orbit effect is generated in the magnetic layer itself, and does not require a distinct high-SOI material next to it; (ii) the spin-orbit field can be either perpendicular or collinear to the magnetization, a configuration that has not been explored in DWP yet due to the lack of strongly uniaxial in-plane metals subject to spin-orbit fields [40].

\section{PHENOMENOLOGY}

We first describe the DW propagation phenomenology under field and current [Figs. 1(c)-1(j)]. After saturation by an external field $H_{\text {sat }}$, a reversed domain was nucleated by a high current pulse. In the narrowest, 2 and $4 \mu \mathrm{m}$ wide tracks, 
DWs required a small field opposite to $H_{\text {sat }}$ to depin. Under this small field only, note that the DWs did not move. In the $10 \mu \mathrm{m}$ track, the pinning of DWs was sufficiently low to enable current-induced motion without external magnetic field. In the $\mathrm{C}_{/ /}$track [see Figs. 1(d)-1(f)], we observed that DWs only depin in the hole current direction, regardless of the DW charge (tail-to-tail or head-to-head), as summarized in Fig. 1(c). Holes flow in the same direction as the conventional current, i.e., opposite the electrons. We will for now call this effect "STT-like", and suppose the DW feels a local field $\vec{H}_{S T T}$ oriented parallel to the easy axis. In the $\mathrm{C}_{\perp}$ tracks, the DW behavior is more complex [see Figs. 1(h) and 1(i)], and seems to consist of two competing effects. It depends on the DW polarity which we label [Fig. $1(\mathrm{~g})] p=+1(p=-1)$ when the magnetization of the first domain crossed by the current is $+\pi / 2(-\pi / 2)$ rotated with respect to the current direction. We observed that regardless of the current and $H_{\text {sat }}$ signs, $p=-1$ DWs alway propagate, whereas $p=+1$ DWs are always pinned, as summarized schematically in Fig. 1(g). This suggests the current creates an effective field $\vec{H}_{\text {eff }}$ pointing in the magnetization direction of the expanding domain, competing with the STT-like contribution. When a field is needed to depin, as in the $2 \mu \mathrm{m}$ wide tracks, an identical phenomenology was observed [images in Fig. 1(i)]. Note that in one particular configuration, DWs occasionally depinned in the direction opposite to the current flow [Fig. 1(j)]. Finally, the reproducibility of these observations was verified in detail between $4 \mathrm{~K}$ and $80 \mathrm{~K}$, as well as the robustness of the phenomenology [41]. These results are in stark contrast with those obtained by transport-only measurements on planar (Ga,Mn)As with biaxial anisotropy. They had shown either no dependence on current polarity [31], or deduced indirectly a DW propagation direction against the hole current [32].

To get an insight on the nature of the torques at play in the DW motion, we then acquired hysteresis cycles on the $2 \mu \mathrm{m}$ wide $\mathrm{C}_{\perp}$ track for different values of a dc current [see Fig. 2(a)]. In order to maintain a constant effective temperature $\left(T_{\text {eff }}=83 \mathrm{~K}\right)$, the temperature rise during sample excitation was carefully characterized and adjusted for by tuning the cryostat temperature (see Appendix A). Figure 2(a) shows that the hysteresis cycles are shifted in opposite directions for positive or negative currents, indicating that the effect of current is equivalent to an effective magnetic field along the easy axis. In order to study this effect, we monitored the depinning field out of a particular defect along the stripe, $H_{\text {dep }}$, as a function of current, and compared it to its value without any current: $\delta H_{\text {dep }}(J)=H_{\text {dep }}(J)-H_{\text {dep }}(J=$ $0)$. Positive and negative dc currents were used, so that the four different relative orientations of current/field were explored [see side schematics in Fig. 2(b)], allowing us to disentangle the two competing effects. They reproduced the blocking/passing configurations observed under pulsed current in Figs. 1(i) and 1(j). In the resulting $\delta H_{\text {dep }}(J)$ plot of Fig. 2(b), $\delta H_{\text {dep }}<0\left(\delta H_{\text {dep }}>0\right)$ implies that the current made it easier (more difficult) to depin the DW. At low current density and in the south-east quadrant of Fig. 2(b), it becomes very difficult to pinpoint precisely how different the depinning field is from the $J=0$ one. As the current density becomes higher, however, we notice that the $\delta H_{\text {dep }}(J)$ data essentially consists of two intersecting lines of positive (negative) slope $\frac{\delta H_{\text {dep }+}}{J}\left(\frac{\delta H_{\text {dep- }}}{J}\right)$. We can therefore extract an effective fieldlike contribution $\frac{\mu_{0} H_{\text {eff }}}{J}=\frac{\mu_{0}}{2}\left(\left|\frac{\delta H_{\text {dep }+}}{J}\right|+\left|\frac{\delta H_{\text {dep }-}}{J}\right|\right)=$ $(3.6 \pm 0.2) \times 10^{-2} \mathrm{mT} / \mathrm{GA} \mathrm{m}^{-2}$ and a domain-independent, STT-like contribution $\frac{\mu_{0} H_{S T T}}{J}=\frac{\mu_{0}}{2}\left(\left|\frac{\delta H_{\text {dep }+}}{J}\right|-\left|\frac{\delta H_{\text {dep }-}}{J}\right|\right)=(6 \pm$ 1) $\times 10^{-3} \mathrm{mT} / \mathrm{GA} \mathrm{m}^{-2}$. We therefore conclude that $\frac{H_{\text {eff }}}{J}$ is six times larger than the STT-like contribution $\frac{H_{S T T}}{J}$, and is of the same order of magnitude as the total spin-orbit field efficiency in Refs. [27-30]. Note that $H_{\text {eff }}$ exhibits the same symmetry as the Oersted field accompanying the passage of the current. Micromagnetic simulations under $J=20 \mathrm{GA} \mathrm{m}^{-2}$ show that the transverse $(y)$ component of the Oersted field is around $\pm 0.2 \mathrm{mT}$ (in the center of the top/bottom interfaces), and its out-of-plane $(z)$ component is $\pm 0.8 \mathrm{mT}$ (at midheight of the track edges). However, the substrate being quite insulating, the current density is mostly confined to the magnetic layer, and we expect the average Oersted field in the track to be close to zero.
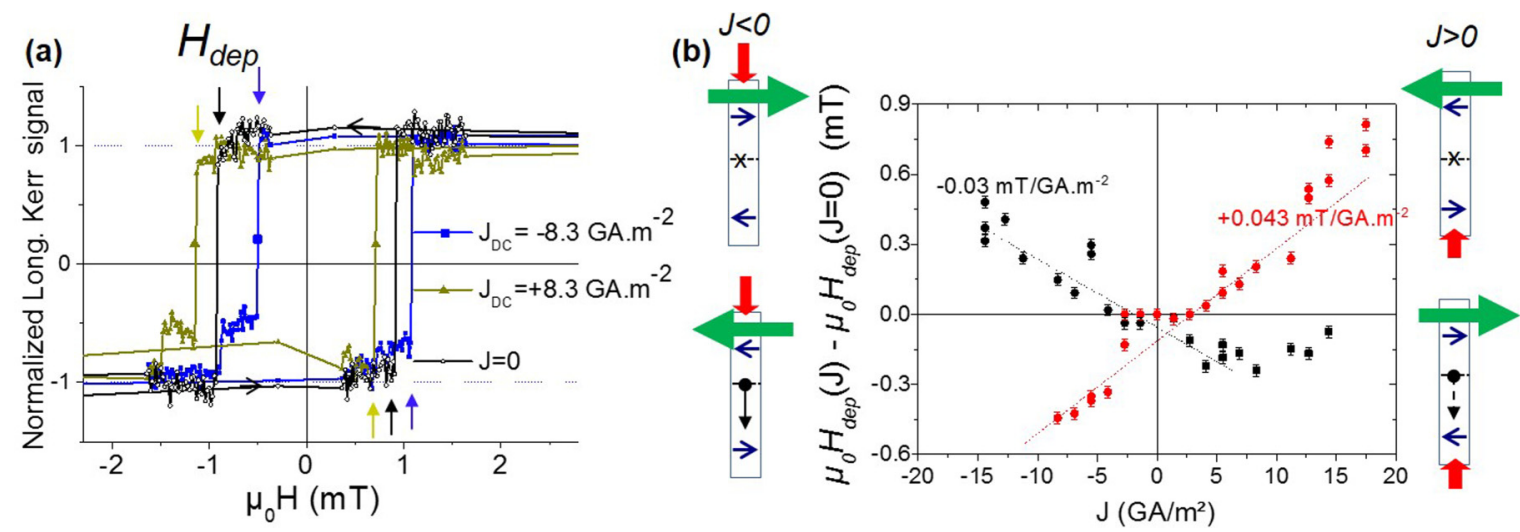

FIG. 2. Estimation of the current-induced STT-like and FL-like contributions in the $2 \mu \mathrm{m}$ wide $\mathrm{C}_{\perp}$ track, at $T_{\text {eff }}=83 \mathrm{~K}$. (a) Normalized hysteresis cycles under continuous current $J= \pm 8.3 \mathrm{GA} \mathrm{m}^{-2}$, and without current. They are obtained by taking longitudinal Kerr images as the field is cycled, and averaging the signal over the entire surface of the track. Hysteresis cycles averaged on the reservoir under current coincide with those averaged on the track without current. (b) The DW depinning field out of a given trap $H_{\text {dep }}(J)$ is monitored as a function of current density and compared to the one without applied current $H_{\text {dep }}(J=0)$. The difference is indicated in the $y$ axis. The four schematics represent the physical situations encountered in the four corresponding quadrants of the plot, with the same arrow legend as in Fig. 1. 

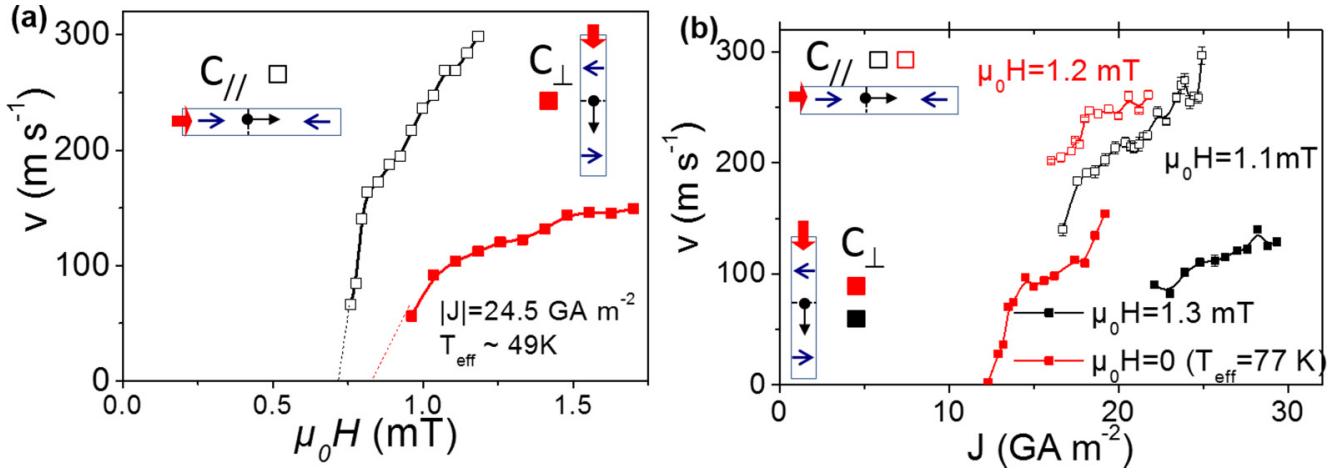

FIG. 3. Domain-wall velocity: (a) velocity versus field curves $\left(\mathrm{C}_{/ /}\right.$and $\mathrm{C}_{\perp} 2 \mu \mathrm{m}$ wide tracks) at constant temperature and current density. (b) Velocity versus current density for tracks $\mathrm{C}_{/ /}\left(2 \mu \mathrm{m}\right.$ wide, $\mu_{0} H=1.1$ and $\left.1.2 \mathrm{mT}\right)$ and $\mathrm{C}_{\perp}\left(2 \mu \mathrm{m}\right.$ wide, $\mu_{0} H=1.3 \mathrm{mT}$, and $10 \mu \mathrm{m}$ wide, no field). Measurements taken at $T_{\text {eff }}=49 \pm 1 \mathrm{~K}$ for the $2 \mu \mathrm{m}$ wide tracks, and at $T_{\text {eff }}=77 \pm 2 \mathrm{~K}$ for the $10 \mu \mathrm{m}$ wide one.

We can now summarize the current-induced DW motion phenomenology in $(\mathrm{Ga}, \mathrm{Mn}) \mathrm{As}$ with uniaxial in-plane anisotropy as follows. When the current flows colinear to the magnetization $\left(\mathrm{C}_{/ /}\right.$tracks), DW motion occurs in the hole current direction regardless of domain charge. This is opposite to the direction observed for spin-transfer torque in perpendicularly magnetized $(\mathrm{Ga}, \mathrm{Mn}) \mathrm{As}$ films. When the current flows perpendicular to the magnetization $\left(\mathrm{C}_{\perp}\right.$ tracks $)$, this STT-like contribution competes with an effect six times larger, an effective transverse field $H_{\text {eff }}$ proportional to the current.

Finally, we performed DW velocity measurements on the $\mathrm{C}_{/ /}$and $\mathrm{C}_{\perp}$ tracks (see Fig. 3). Displacements under increasing current pulse lengths were obtained as described in Appendix B. Figure 3(a) shows $v_{J}(H)$ curves displaying the DW velocity as a function of the applied magnetic field, in the presence of current pulses of constant amplitude. The maximum measurable velocity is determined by the track length. After a depinning regime at low fields, the DW velocity increases linearly with field, reaching up to $300 \mathrm{~m} \mathrm{~s}^{-1}$ on the $2 \mu \mathrm{m}$ wide $\mathrm{C}_{/ /}$track. These velocities are typical of those measured under field only on a very similar nonpatterned layer [39], and result from the large DW width of in-plane (Ga,Mn)As. In the $\mathrm{C}_{\perp}$ tracks, velocities are overall smaller [up to $150 \mathrm{~m} \mathrm{~s}^{-1}$, Fig. 3(a)]. Comparing $\mathrm{C}_{/ /}$and $\mathrm{C}_{\perp} v_{J}(H)$ curves taken at the same current density shows that the $\frac{\partial v}{\partial H}$ mobilities lie in a ratio of 4:1 (measured after the depinning regime). In the stationary regime, we expect field mobilities to vary in a first approximation like the static DW width, estimated by micromagnetic simulations [Fig. 4(a)] to be almost three times larger in the charged DWs of $C_{/ /}$tracks $\left(\Delta_{0}=40\right.$ $\mathrm{nm})$, than in the uncharged DWs of $\mathrm{C}_{\perp}\left(\Delta_{0}=15 \mathrm{~nm}\right)$. The measured mobility ratio is therefore a signature of the stationary regime. Also, the precessional regime is expected to be preceded by a large velocity plateau, seen under field only on unpatterned samples [39] and in micromagnetic simulations for $\mathrm{C}_{\perp}$ and $\mathrm{C}_{/ /}$tracks [Fig. 4(b)]. This plateau was not observed experimentally under applied field/current.

The field was then kept constant $(1.1,1.2,1.3$, or $0 \mathrm{mT})$, and current pulses $\left(J=7-25 \mathrm{GA} \mathrm{m}^{-2}\right)$ were applied at constant effective temperature. This generated $v_{H}(J)$ curves [Fig. 3(b)]. Once more, velocities of up to $300 \mathrm{~m} \mathrm{~s}^{-1}$ were observed on the $2 \mu \mathrm{m}$ wide $\mathrm{C}_{/ /}$track, and up to $150 \mathrm{~m} \mathrm{~s}^{-1}$ in $\mathrm{C}_{\perp}$ tracks, again pointing to the stationary regime. The resulting current mobility $\frac{\partial v}{\partial J}$ is $11 \pm 1 \mathrm{~mm}^{3} \mathrm{C}^{-1}$ for the $2 \mu \mathrm{m}$ wide $\mathrm{C}_{/ /}$ tracks $\left(T_{\text {eff }}=49 \mathrm{~K}\right)$ and $10 \mu \mathrm{m}$ wide $C_{\perp}$ track $\left(T_{\text {eff }}=77 \mathrm{~K}\right)$, over ten times larger than the mobilities measured on outof-plane magnetized (Ga,Mn)As [34-37]. No field assistance was required for the wider $10 \mu \mathrm{m} \mathrm{C}_{\perp}$ track [Fig. 3(b)], on which creep motion was also observed at low current densities ( $J=7 \mathrm{GA} \mathrm{m}^{-2}$, velocities too small to appear on the curve).

(a)
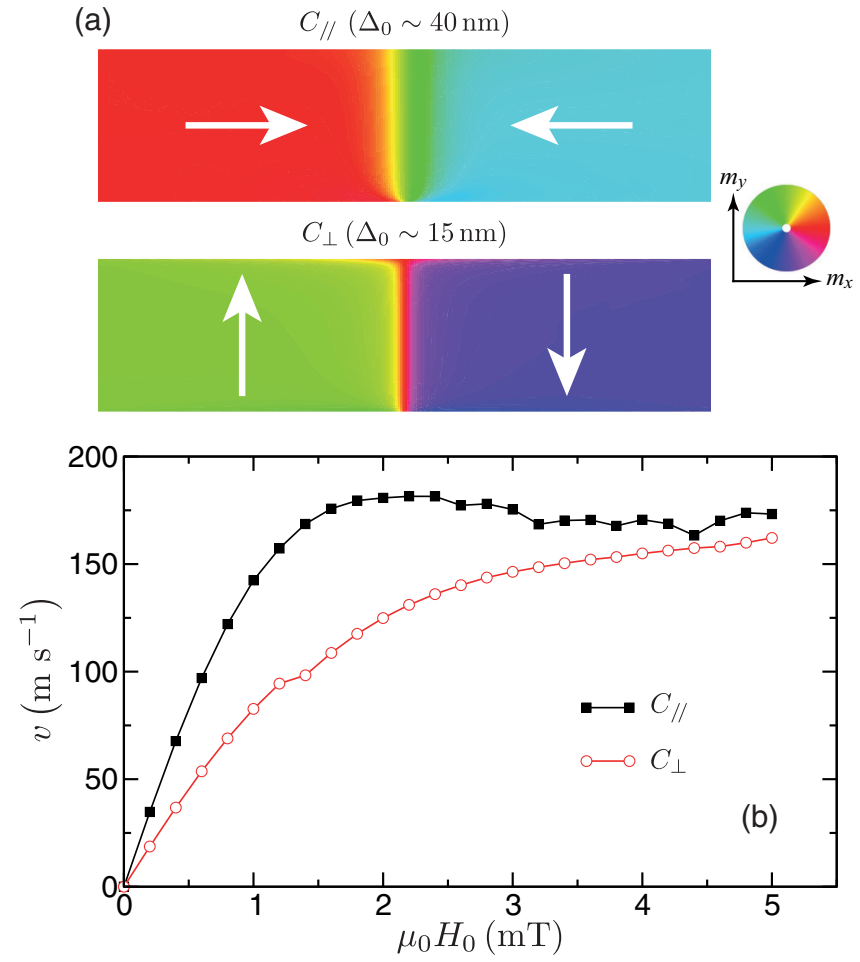

FIG. 4. Micromagnetic simulations $(T=60 \mathrm{~K}$ micromagnetic parameters of the sample, and $T=0$ in mumax code [42]) for both track configurations. (a) Static domain wall width. Smaller DW widths translate into smaller mobilities under field. (b) Field-driven DW propagation, subtracting surface magnetic charges at wire ends to simulate infinite wire. The best agreement with measurements on unpatterned samples [39] was obtained with $\alpha=0.025$. The precessional regime is reached at a few $\mathrm{mT}$, in the plateau of the curve. 
In the $2 \mu \mathrm{m} \mathrm{C}_{\perp}$ track, a lower mobility of $6 \pm 1 \mathrm{~mm}^{3} \mathrm{C}^{-1}$ was measured.

\section{POSSIBLE TORQUES AT PLAY}

To make sense of these unexpected results, we begin by considering two types of current-induced torques: torques that push DWs unidirectionally regardless of their polarity or charge, which will be named STT-like, or torques driven by the Rashba and Dresselhaus spin-orbit effective fields.

\section{A. Spin-orbit effective field torques}

Dresselhaus and Rashba terms in strained ( $\mathrm{Ga}, \mathrm{Mn}) \mathrm{As}$ induce a total effective field $\vec{H}_{s o}$ proportional to the current density and transverse to the track direction [Figs. 1(a) and 1(b)], expected to be slightly larger in $C_{\perp}$ than in $\mathrm{C}_{/ /}$tracks [27-30,43]. Similar to what has been calculated and observed in metals [7,44-47], this field can act on the magnetization via two torques: a fieldlike torque [48] (FL-SOT) $\vec{H}_{s o} \times \vec{M}$ or a Slonczweski-like torque (SL-SOT) $\vec{M} \times \vec{H}_{S L}$ with $\vec{H}_{S L} \propto \vec{M} \times \vec{H}_{s o}$. The FL-SOT is sensitive to the charge or polarity of the DW, but not to its chirality (magnetization orientation in the DW), while the SL-SOT is sensitive to the magnetization configuration within the DW (Bloch/Néel, chirality). Both torques have been calculated [26,43] and measured [27-30,43] in monodomain $(\mathrm{Ga}, \mathrm{Mn}) \mathrm{As}$ and $(\mathrm{Ga}, \mathrm{Mn})(\mathrm{As}, \mathrm{P})$ and found to be of the same order of magnitude [43].

We have summarized schematically their expected effect on DWs of $\mathrm{C}_{/ /}$and $\mathrm{C}_{\perp}$ tracks in Fig. 5. In $\mathrm{C}_{/ /}$tracks, the FL-SOT simply stabilizes a Néel DW structure against Walker breakdown, and possibly imposes a DW chirality during its creation [Fig. 5(a)]. In $\mathrm{C}_{\perp}$ tracks however, $\vec{H}_{s o}$ is collinear to the domain magnetization, so will act like the effective field $\vec{H}_{\text {eff }}$ evidenced earlier. Hence we suggest $\mu_{0} \vec{H}_{\text {eff }}=\mu_{0} \vec{H}_{S O}$. We established above that at $83 \mathrm{~K}$ it varies with current as $3.6 \times 10^{-2} \mathrm{mT} / \mathrm{GA} \mathrm{m}^{-2}$ [Fig. 2(b)], close to the (2.0-10.6) $\times$ $10^{-2} \mathrm{mT} / \mathrm{GA} \mathrm{m}^{-2} \mathrm{SO}$ field efficiencies found by other authors
[27-30] for $J / /[110]$. This yields for our typical current densities (e.g., $J=20 \mathrm{GA} \mathrm{m}^{-2}$ ) $\mu_{0} H_{S O}=0.4-2.7 \mathrm{mT}$, very much of the order of the applied static fields. Its direction is represented in Figs. 1(g) and 5(b), 5(d) by a green hollow arrow.

Note that the total spin-orbit field we find for $J / /[110]$ is of opposite sign to the one found in previous studies of in-plane magnetized (Ga,Mn)As devices [27-30,43]. These measurements were done on samples quite similar to ours in Mn content, Curie temperature, of varying anisotropy (uniaxial or biaxial), and monodomain or not. A notable difference would be our sample thickness $(50 \mathrm{~nm})$, e.g., twice that of the thickest of these studies $(25 \mathrm{~nm})$. One could tentatively say that a thicker layer would reduce the Rashba (interfacial) contribution to the total $\vec{H}_{\text {so }}$ compared to thinner layers. However, since it is unclear what governs the sign of the Rashba field, and how exactly it varies from layer to layer, it is very difficult to infer anything from this observation. A more flagrant discrepancy is that the studies of Refs. [27-30] are all based on magnetotransport measurements, whereas we proceed via a direct visualization of domains. However, with a correct characterization of both hole current and applied field directions, this should not affect the sign of $\mu_{0} H_{S O}$. The reason for this sign difference therefore remains elusive for the moment.

The SL-SOT was proposed to explain similar intriguing DW propagation direction and velocities in metals [49]. We label it "efficient" (propagation in the stationary regime) when it tilts the magnetization out of the plane of rotation of the DW (materialized by dotted lines in Fig. 5), and "inefficient" (propagation in the precessional regime only) when it merely rotates the DW magnetization. As represented schematically in Figs. 5(a) and 5(b), the SL-SOT will be inefficient for $\mathrm{C}_{/ /}$and $\mathrm{C}_{\perp}$. We also consider DW structures other than the Néel or transverse ones [50]. The SL-SOT can then induce efficient propagation for $\mathrm{C}_{/ /}$tracks provided a significant Bloch component is present [Fig. 5(c)]. For the resulting DW propagation direction to be independent of the DW charge, as observed experimentally [Figs. 1(c)-1(f)],

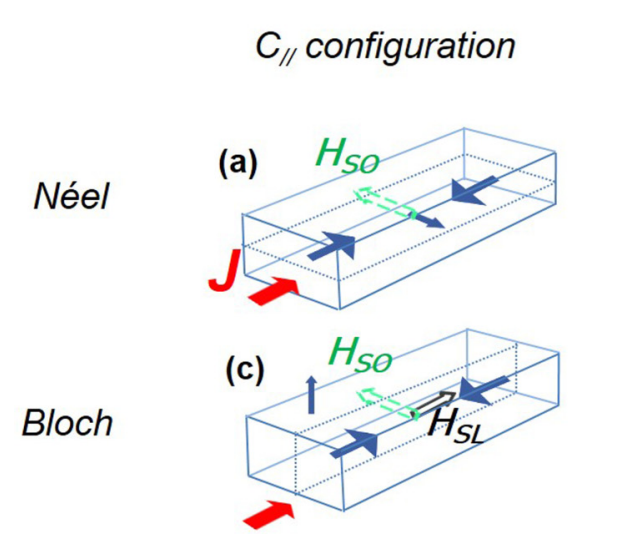

\section{$C_{\perp}$ configuration}
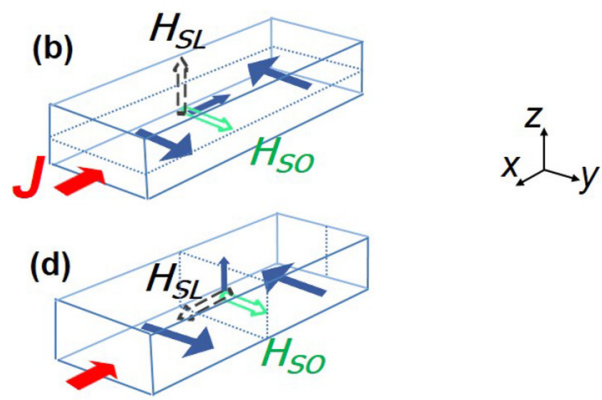

FIG. 5. Effective fields acting on the magnetization (blue arrows) involved in the fieldlike torque (green hollowed arrows) and the Slonczweski-like torque (black hollowed arrows), $\vec{H}_{S L} \propto \vec{H}_{s o} \times \vec{M}$. Their effect on $\mathrm{C}_{/ /}$(a),(c) and $\mathrm{C}_{\perp}$ (b), (d) configurations, and supposing Néel (a),(b) or Bloch (c),(d) domain walls are represented using dashed contours if the field is only efficient in the precessional regime, or continuous contours if it is efficient in the stationary regime. The direction of the hole current is indicated by a red arrow and the dotted contours materialize the plane of rotation of the magnetization in the domain wall. The direction of $\vec{H}_{s o}$ has only been inferred experimentally in (b). 
DWs would however need to be chiral, meaning that the magnetization would for instance need to point "up" in headto-head DWs, and "down" in tail-to-tail DWs. This property usually accompanies the DMI [16], a point that would require further theoretical development for the case of ( $\mathrm{Ga}, \mathrm{Mn}) \mathrm{As}$. This torque would however be inefficient in the $\mathrm{C}_{\perp}$ tracks [Fig. 5(d)].

To summarize, Rashba and Dresselhaus spin-orbit effective fields could only explain the fieldlike torque observed in $\mathrm{C}_{\perp}$ tracks. To make sense of the STT-like effects with a torque involving an effective field proportional to the current, one would need it to be along $z$, instead of in-plane and transverse to the track. Under the form of a Slonzweski-like torque $\vec{M} \times$ $\vec{H}_{\text {eff }, z} \times \vec{M}$, it could indeed push DWs efficiently along the hole current on both types of tracks, provided it had the correct sign and DWs were chiral. Such a field has recently been evidenced in monodomain $\mathrm{Ta} / \mathrm{CoFeB} / \mathrm{TaOx}$ trilayers [51]. It is equivalent to an in-plane electric field perpendicular to the track, and proportional to the current density. In Yu et al. [51] it originated from the lateral structural asymmetry induced by the wedged cross section of their sample, but its origin in our case would be unclear. It would also be problematic to reconcile it with current-induced domain wall propagation in out-of-plane magnetized $(\mathrm{Ga}, \mathrm{Mn}) \mathrm{As}$ and $(\mathrm{Ga}, \mathrm{Mn})(\mathrm{As}, \mathrm{P})$ : a $\vec{H}_{\text {eff }, z}$ field would prevent consecutive DWs from shifting synchronously under current, as has been observed in these samples [35-37].

To conclude on this part on spin-orbit effective fields, we wish to comment on an implicit hypothesis made in our approach. Here the spin-orbit field $\vec{H}_{s o}$ is assumed to be only weakly affected by the presence of a domain wall [as represented schematically in Figs. 1(c) and 1(g)]. However, domain walls could be the locus of significant $\mathrm{Mn}$ (and therefore hole) depolarization [20,52], which could in turn modify the local spin-orbit field amplitude. $\vec{H}_{s o}$ has so far mainly been measured in monodomain samples, and for current/magnetization on high symmetry axes [27,29]. Let us mention however that $\mathrm{Li}$ et al. [30] have measured $\vec{H}_{s o}$ on $10 \mu \mathrm{m}$ wide devices probably accommodating DWs, and found it to be similar to those of the $80 \mathrm{~nm}$ wide monodomain devices of Fang et al. [29] along [1 \pm 10$],[100]$, which supports our initial hypothesis. Kurebayashi et al. [43] moreover studied, on a monodomain sample, the dependence of the SL field $\vec{H}_{z} \propto \vec{H}_{s o} \times \vec{M}$ on the angle $(\vec{M}, \vec{J})$, and evidenced weak anisotropic effects.

\section{B. Spin-transfer-like torques}

We now consider the effect of STT-like torques, and focus on the generic "nonadiabatic" term $\beta \vec{M} \times[(\vec{J} \cdot \vec{\nabla}) \vec{M}]$, where $J$ is the current density and $\beta$ is a phenomenological factor related to spin accumulation at the domain wall. In the stationary regime, a velocity proportional to the current density is expected, with $v \propto \frac{\beta}{\alpha} J, \alpha$ being the Gilbert damping term.

Previous work [35-37] done on 25-50 nm thick out-ofplane magnetized $(\mathrm{Ga}, \mathrm{Mn}) \mathrm{As}$ or $(\mathrm{Ga}, \mathrm{Mn})(\mathrm{As}, \mathrm{P})$ has evidenced a negative mobility of DWs driven by current. Assuming a spin-relaxation transfer torque [3], $\frac{\partial v}{\partial J}=\frac{\beta_{s r}}{\alpha} \frac{\left|P_{c}\right| \mu_{B}}{e M_{s}}<0$ was then justified by the negative carrier polarization $P_{c}$ arising from the antiferromagnetic Mn spin/hole spin interaction, and a value of $\beta_{s r} / \alpha \approx-1.0 \pm 0.5$ was found [53]. The positive mobilities measured on both $C_{/ /}$and $\mathrm{C}_{\perp}$ tracks, however, suggest that in planar $(\mathrm{Ga}, \mathrm{Mn}) \mathrm{As}$ this effect is in fact dominated by a counterpropagating one. From the velocity curves [Fig. 3(b)], we estimate $\beta / \alpha \approx 12$ (see numerical details in Appendix $\mathrm{C}$ ) for the $\mathrm{C}_{/ /}$tracks. Assuming $\beta_{s r} / \alpha \approx$ -1 implies that an STT-like mechanism of opposite sign needs to account for the remaining 13. Note that the spin-relaxation transfer torque is very probably present though, since the measured $M_{s}(T)$ curve exhibits a very standard shape, which is consistent with the efficient mutual polarization of the Mn and hole spin populations. This contrasts with ultrathin metallic films sandwiched between other layers, which end up being poorly spin polarized due to their weak relative conductivity in the stack $[11,13]$. In $\mathrm{C}_{\perp}$ tracks a lower ratio of $\beta / \alpha<+0.7-5$ was estimated from both the velocity curves and the hysteresis cycles taken under dc current (Fig. 2 and Appendix C).

The term $\beta$ phenomenologically accounts for many different microscopic phenomena leading to spin relaxation such as spin-flip scattering or DW-induced relaxation [4,54]. In metals it also covers the appearance of a DW resistance at abrupt interfaces [55-58], leading to a momentum transfer force [7,59-62] never clearly identified experimentally [63]. Two contributions have been identified in the spin-relaxation nonadiabatic torque. The first one is "interband" and results from the modification of the electron wave functions under an applied electric field [21,22,43,54]. It is weakly affected by the (Ga,Mn)As KL SOI. The second one is "intraband" and reflects the modification of the band populations by the electric field, via the Fermi-Dirac coefficient. In (Ga,Mn)As, the "interband" component dominates as a result of the strong SOI, with predicted $[20,21] \beta / \alpha \approx 10$. In particular, it can overcome the intrinsic limited efficiency of a total momentum-conserving torque transfering exactly $\hbar$ between conduction carriers and local magnetic moments. Interestingly, Garate et al. [21] predict a sign opposite to the traditional adiabatic STT in certain cases for the intraband component. The large effective $\beta / \alpha$ observed in our in-plane tracks makes this torque the most likely mechanism at work. The influence of the anisotropy and of the domain-wall width having not been addressed yet in these calculations, the reason why this contribution would be absent in out-of-plane magnetized (Ga,Mn)As remains elusive. We have calculated $k$-space maps of the spin and orbital components $S_{z}$ and $L_{z}$ by $\vec{k} \cdot \vec{p}$ theory for in-plane and out-of-plane magnetized (Ga,Mn)As, and have not found any noteworthy differences. In particular, the calculated hole polarization at the Fermi energy is identical. Instead, a possibility to be explored is the influence of the domain-wall width. Indeed we do observe that the high positive mobility under current seems to decrease with domain-wall width: $\beta / \alpha \approx+12$ for $\Delta \approx 40 \mathrm{~nm}\left[\mathrm{C}_{/ /}\right.$tracks, Fig. $\left.4(\mathrm{a})\right], \beta / \alpha<$ $+0.7-5$ for $\Delta \approx 15 \mathrm{~nm}\left(\mathrm{C}_{\perp}\right.$ tracks $)$, and finally $\beta / \alpha \approx-1$ for $\Delta \approx 5 \mathrm{~nm}$ [out-of-plane magnetized (Ga,Mn)As]. Although this agrees with the tendency of the SOI-induced torques to increase with domain-wall width calculated in ballistic nickel domain walls [58], it disagrees with the prediction of Nguyen et al. [20].

At this stage, based on this phenomenological study and to reconcile all current-induced observations in (Ga,Mn)As/(Ga,Mn)(As,P), one can infer that the KL SOI 
spin transfer torque is responsible for DW propagation along the hole current in in-plane layers with wide domain walls, but is absent in out-of-plane layers. The domain-wall width is however clearly not the only relevant parameter, since De Ranieri et al. [37] have observed current-induced DWP opposite the hole current on perpendicularly magnetized $(\mathrm{Ga}, \mathrm{Mn})(\mathrm{As}, \mathrm{P})$ tracks with Néel DWs. Further theoretical work on the domain-wall width and anisotropy dependence of this torque would therefore greatly enrich this discussion.

\section{Spin Hall and anomalous Hall effects torques}

Finally, we mention some of the other effects likely to affect current-driven DWP. Among them, the spin Hall effect [64] torque that appears when a ferromagnet is adjacent to a nonmagnetic high spin-orbit coupling metal has proved decisive to explain propagation against the electron flow in ultrathin metallic layers [10-14]. It is however very unlikely to exist in the bulk of a ferromagnet. One could argue that if current leaked into the GaAs substrate, the presence of the spin-asymmetric vacuum/(Ga,Mn)As/GaAs interfaces could in theory pump a spin current perpendicular to the layer. The scaling of the tracks' resistance with their width however points to a sufficiently insulating substrate to neglect this contribution. Closely related to it, the anomalous Hall effect is large in $(\mathrm{Ga}, \mathrm{Mn}) \mathrm{As}$. It was recently shown both theoretically and experimentally to be a possible source of large $\beta / \alpha$ ratio in Permalloy $[65,66]$. This effect however seems limited to vortex domain walls.

\section{CONCLUSIONS}

We have observed current-induced domain-wall propagation in uniaxial in-plane (Ga,Mn)As tracks. The currentdependent DW mobility is up to ten times higher and of opposite sign than in out-of-plane magnetized (Ga,Mn)As and cannot be explained by the arguments put forward in metallic structures, where similar effects were evidenced. In particular, the fieldlike and Slonczweski-like torques associated with the Rashba/Dresselhaus spin-orbit fields alone cannot account for these observations. The existence of an efficient Kohn-Luttinger SOI spin-transfer mechanism, overshadowing the usual spin-relaxation channel seems so far the most likely candidate, with the constraint that it would need to be much stronger in in-plane than in out-ofplane (Ga,Mn)As/(Ga,Mn)(As,P) layers. This work, however, provides a strong motivation to further study in-plane magnetized $(\mathrm{Ga}, \mathrm{Mn}) \mathrm{As}$ tracks, and more generally to engineer uniaxial in-plane materials showing strong intrinsic spinorbit interactions. This should allow one to explore the possibility of obtaining these high mobilities without the requirement of a pure-spin current source like in metallic heterostructures.

\section{ACKNOWLEDGMENTS}

We thank A. Thiaville for his continuous interest in this work, as well as V. Cros, J. Sampaio, and A. Manchon for stimulating discussions. We acknowledge M. Bernard, S. Majrab, and M. Rostiche for their technical assistance, and
J. von Bardeleben for the cavity-FMR measurements. This work has been supported by the French RENATECH network.

\section{APPENDIX A: ESTIMATION OF THE EFFECTIVE TEMPERATURE}

As routinely done on $(\mathrm{Ga}, \mathrm{Mn})(\mathrm{As}, \mathrm{P})$ and $(\mathrm{Ga}, \mathrm{Mn}) \mathrm{As}$ samples [35-37], the strong temperature dependence of the resistance $R$ was used to evaluate the effective temperature of the tracks. After taking a calibration $R(T)$ curve under very low current, the cryostat was set at a given temperature. A constant voltage $U$ was then applied, and the track resistance $R$ measured. This yielded a $R(P)$ curve where $P=U^{2} / R$. Using the low current $R(T)$ calibration curve, this was turned into a $T_{\text {stat }}(P)$ curve where $T_{\text {stat }}$ is the track temperature in the stationary regime. In a $1 \mathrm{D}$ heat diffusion model, this linear relationship depends solely on the track dimensions, the substrate thickness, and the thermal conductivity $K$ [67]. We could therefore extract experimentally an effective value of $K$ for the different tracks, and from different starting temperatures $T_{0}$. Typical values of $K=100-150 \mathrm{~W} \mathrm{~m}^{-1} \mathrm{~K}^{-1}$ were obtained. This in turn was used to estimate the effective temperature $\left(T_{\text {eff }}\right)$ after short current pulses, using the specific heat from Ref. [68] (e.g., $C=77 \mathrm{~J} \mathrm{~kg}^{-1} \mathrm{~K}^{-1}$ around $50 \mathrm{~K})$ :

$$
\begin{gathered}
\Delta T(\tau)=\frac{P}{2 \pi K l} \ln \left(\frac{16 D \tau}{w^{2}}\right), \\
\Delta T(\tau \rightarrow \infty)=\frac{P}{\pi K l}\left[\frac{3}{2}+\ln \left(\frac{2 L}{w}\right)\right], \\
T_{\text {stat }}^{\text {eff }}=T_{0}+\Delta T,
\end{gathered}
$$

where $w$ and $l$ are respectively the track width and length, $L=350 \mu \mathrm{m}$ is the substrate thickness, $D=K / \rho C$ is the heat diffusion coefficient, and $\rho$ is the mass density.

\section{APPENDIX B: METHODOLOGY OF DW VELOCITY MEASUREMENT}

The procedure for image acquisition was identical to the one used in Ref. [39]. An image was first taken in zero field, after saturating the sample $\left(\mu_{0} H_{\text {sat }}= \pm 8 \mathrm{mT}\right)$. Consecutive images (after field/current application) were divided by this reference image in order to enhance the domain contrast. Different DW propagation behaviors_-pinned, depinning, and depinned regimes-were observed depending on the value of current/field [Fig. 6(a)]. Given the high velocity of the DWs, short pulses were required. The velocity was obtained as the slope of the averaged displacements versus pulse length $\tau$ [Fig. 6(b)]. For each $\tau$, several acquisitions were made, giving a distribution of displacements.

\section{APPENDIX C: EXPERIMENTAL ESTIMATION OF $\beta$}

This was done using either the velocity curves taken under pulsed current [Fig. 3(b)], or the hysteresis cycles taken under dc current (Fig. 2). The magnetization at saturation was determined by SQUID: $M_{s}=33 \mathrm{kA} / \mathrm{m}$ at $49 \mathrm{~K}$ and $M_{s}=$ $16 \mathrm{kA} / \mathrm{m}$ at $77 \mathrm{~K}$. The domain wall widths were taken as $15 \mathrm{~nm}(40 \mathrm{~nm})$ for $\mathrm{C}_{\perp}\left(\mathrm{C}_{/ /}\right)$tracks (Fig. 4). 

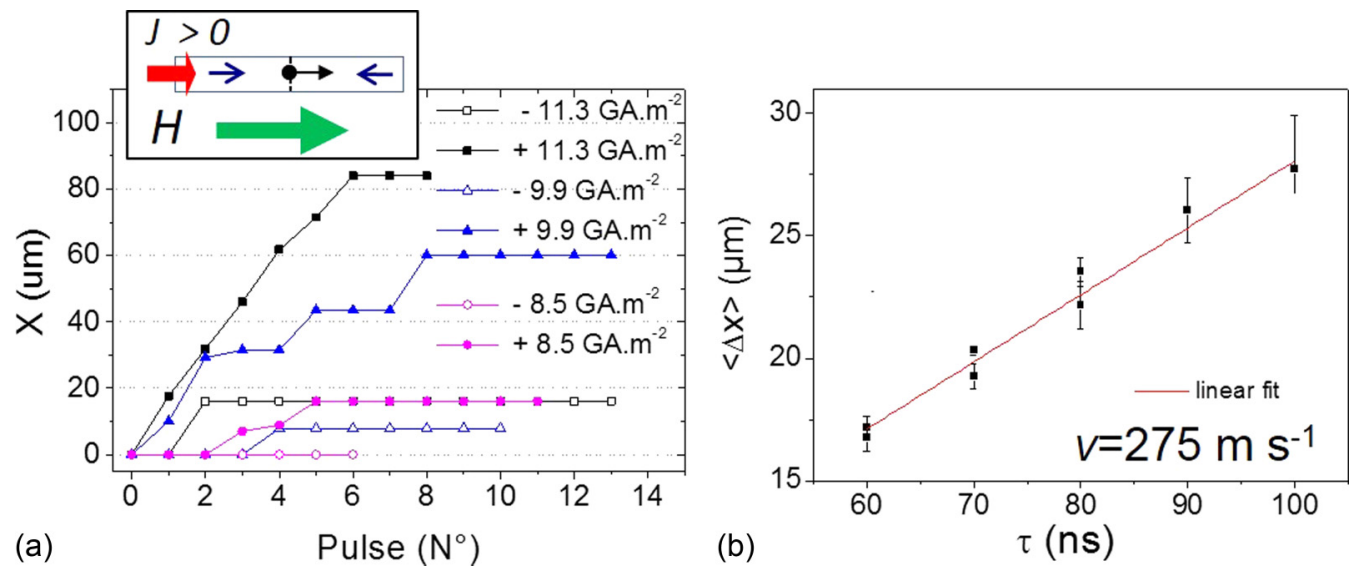

FIG. 6. $2 \mu \mathrm{m}$ wide $\mathrm{C}_{/ /}$tracks: (a) position of the DW versus current pulse number at fixed applied field $\mu_{0} H=0.7 \mathrm{mT}$ and $T_{\text {eff }} \approx 62 \mathrm{~K}$, pulse duration $\tau=100$ ns. Pinned (magenta), depinning (blue), and depinned (black) regimes are clearly identified for increasing current densities. No prlopagation or depinning are observed for negative values of the current. (b) Domain wall velocity determination from the averaged domain-wall displacement, at fixed applied field $\mu_{0} H=1.3 \mathrm{mT}, J=21.75 \mathrm{GA} \mathrm{m}^{-2}$, and $T_{\text {eff }} \approx 49 \mathrm{~K}$.

From the velocity curves: in the stationary regime, the velocity is given by $\frac{\partial v}{\partial J}=\frac{\beta}{\alpha} \frac{P_{c} \mu_{B}}{e M_{s}}$ for the $\mathrm{C}_{/ /}$tracks, and $\frac{\partial v}{\partial J}=\frac{\beta_{s s}}{\alpha} \frac{P_{c} \mu_{B}}{e M_{s}}+\frac{\gamma \Delta}{\alpha} \frac{\partial \mu_{0} H_{S O}}{\partial J}$ for the $\mathrm{C}_{\perp}$ tracks. $\frac{\partial \mu_{0} H_{S O}}{\partial J}=3.6 \times$ $10^{-2} \mathrm{mT} / \mathrm{GA} \mathrm{m}^{-2}$ was determined experimentally from the hysteresis cycles at $83 \mathrm{~K}$ (Fig. 2). For lack of lower temperature measurement, we will take this as a lower boundary of the 49 and $77 \mathrm{~K} \frac{\partial \mu_{0} H_{S O}}{\partial J}$ values, giving an upper boundary of $\beta / \alpha$. The polarization of holes at the Fermi energy $P_{c}$ was calculated by $k . p$ theory with a hole density of $p=3 \times 10^{20} \mathrm{~cm}^{-3}$ : $\left|P_{c}\right|=0.53$ at $49 \mathrm{~K}$ and $\left|P_{c}\right|=0.4$ at $77 \mathrm{~K}$.
From the hysteresis cycles: converting the STT-like contribution into a value of $\beta$ with $\frac{\mu_{0} H_{S T T}}{J}=\frac{\beta}{\gamma \Delta}$, following Ref. [69].

For $\mathrm{C}_{/ /}$tracks: at $T_{\text {eff }}=49 \mathrm{~K}$, the mobility of the $2 \mu \mathrm{m}$ wide track $\frac{\partial v}{\partial J}=+11.1 \pm 0.5 \mathrm{~mm}^{3} \mathrm{C}^{-1}$ leads to $\beta / \alpha \approx 12$.

For $\mathrm{C}_{\perp}$ tracks: at $T_{\mathrm{eff}}=49 \mathrm{~K}$, the mobility of the $2 \mu \mathrm{m}$ wide track $\frac{\partial v}{\partial J}=+5.7 \mathrm{~mm}^{3} \mathrm{C}^{-1}$ leads to $\beta / \alpha<2$. At $T_{\text {eff }}=77 \mathrm{~K}$, the mobility of the $10 \mu \mathrm{m}$ wide track $\frac{\partial v}{\partial J}=+11.1 \mathrm{~mm}^{3} \mathrm{C}^{-1}$ leads to $\beta / \alpha<5$. At $T_{\text {eff }}=83 \mathrm{~K}$, the STT-like contribution found by the hysteresis cycles $\frac{\mu_{0} H_{S T T}}{J}=$ $+6.5 \times 10^{-3} \mathrm{mT} / \mathrm{GA} \mathrm{m}^{-2}$ leads to $\beta / \alpha \approx 0.7$.
[1] S. S. P. Parkin and S.-H. Yang, Nat. Nanotechnol. 10, 195 (2015).

[2] A. Chanthbouala, R. Matsumoto, J. Grollier, V. Cros, A. Anane, A. Fert, A. V. Khvalkovskiy, K. A. Zvezdin, K. Nishimura, Y. Nagamine, H. Maehara, K. Tsunekawa, A. Fukushima, and S. Yuasa, Nat. Phys. 7, 626 (2011).

[3] A. Thiaville, Y. Nakatani, J. Miltat, and Y. Suzuki, Europhys. Lett. (EPL) 69, 990 (2005).

[4] S. Zhang and Z. Li, Phys. Rev. Lett. 93, 127204 (2004).

[5] C. Burrowes, A. P. Mihai, D. Ravelosona, J.-V. Kim, C. Chappert, L. Vila, A. Marty, Y. Samson, F. Garcia-Sanchez, L. D. Buda-Prejbeanu, I. Tudosa, E. E. Fullerton, and J.-P. Attané, Nat. Phys. 6, 17 (2009).

[6] T. Koyama, D. Chiba, K. Ueda, K. Kondou, H. Tanigawa, S. Fukami, T. Suzuki, N. Ohshima, N. Ishiwata, Y. Nakatani, K. Kobayashi, and T. Ono, Nat. Mater. 10, 194 (2011).

[7] G. Tatara, H. Kohno, and J. Shibata, Phys. Rep. 468, 213 (2008).

[8] A. Brataas and K. M. D. Hals, Nat. Nanotechnol. 9, 86 (2014).

[9] P. Gambardella and I. M. Miron, Philos. Trans. R. Soc., A 369, 3175 (2011).

[10] R. Lavrijsen, P. P. J. Haazen, E. Muré, J. H. Franken, J. T. Kohlhepp, H. J. M. Swagten, and B. Koopmans, Appl. Phys. Lett. 100, 262408 (2012)

[11] S. Emori, U. Bauer, S.-M. Ahn, E. Martinez, and G. S. D. Beach, Nat. Mater. 12, 611 (2013).
[12] K.-S. Ryu, L. Thomas, S.-H. Yang, and S. S. P. Parkin, Nat. Nanotechnol. 8, 527 (2013).

[13] I. M. Miron, T. Moore, H. Szambolics, L. D. Buda-Prejbeanu, S. Auffret, B. Rodmacq, S. Pizzini, J. Vogel, M. Bonfim, A. Schuhl, and G. Gaudin, Nat. Mater. 10, 419 (2011).

[14] J. Torrejon, F. Garcia-Sanchez, T. Taniguchi, J. Sinha, S. Mitani, J.-V. Kim, and M. Hayashi, Phys. Rev. B 91, 214434 (2015).

[15] A. V. Khvalkovskiy, V. Cros, D. Apalkov, V. Nikitin, M. Krounbi, K. A. Zvezdin, A. Anane, J. Grollier, and A. Fert, Phys. Rev. B 87, 020402(R) (2013).

[16] A. Thiaville, S. Rohart, É. Jué, V. Cros, and A. Fert, Europhys. Lett. (Europhysics Letters) 100, 57002 (2012).

[17] T. Ha Pham, J. Vogel, J. Sampaio, M. Vaňatka, J.-C. RojasSánchez, M. Bonfim, D. S. Chaves, F. Choueikani, P. Ohresser, E. Otero, A. Thiaville, and S. Pizzini, Europhys. Lett. (Europhysics Letters) 113, 67001 (2016).

[18] R. Winkler, Spin-Orbit Coupling Effects in Two-Dimensional Electron and Hole Systems, Springer Tracts in Modern Physics Vol. 191 (Springer, Heidelberg, 2003).

[19] M. Birowska, C. Śliwa, J. A. Majewski, and T. Dietl, Phys. Rev. Lett. 108, 237203 (2012).

[20] A. K. Nguyen, H. J. Skadsem, and A. Brataas, Phys. Rev. Lett. 98, 146602 (2007).

[21] I. Garate, K. Gilmore, M. D. Stiles, and A. H. MacDonald, Phys. Rev. B 79, 104416 (2009). 
[22] D. Culcer, M. E. Lucassen, R. A. Duine, and R. Winkler, Phys. Rev. B 79, 155208 (2009).

[23] B. A. Bernevig and O. Vafek, Phys. Rev. B 72, 033203 (2005).

[24] I. Garate and A. H. MacDonald, Phys. Rev. B 80, 134403 (2009).

[25] K. M. D. Hals, A. Brataas, and Y. Tserkovnyak, Europhys. Lett. (Europhysics Letters) 90, 47002 (2010).

[26] H. Li, H. Gao, L. P. Zârbo, K. Výborný, X. Wang, I. Garate, F. Doğan, A. Čejchan, J. Sinova, T. Jungwirth, and A. Manchon, Phys. Rev. B 91, 134402 (2015).

[27] A. Chernyshov, M. Overby, X. Liu, J. K. Furdyna, Y. LyandaGeller, and L. P. Rokhinson, Nat. Phys. 5, 656 (2009).

[28] M. Endo, F. Matsukura, and H. Ohno, Appl. Phys. Lett. 97, 222501 (2010).

[29] D. Fang, H. Kurebayashi, J. Wunderlich, K. Výborný, L. P. Zârbo, R. P. Campion, A. Casiraghi, B. L. Gallagher, T. Jungwirth, and A. J. Ferguson, Nat. Nanotechnol. 6, 413 (2011).

[30] Y. Li, Y. F. Cao, G. N. Wei, Y. Li, Y. Ji, K. Y. Wang, K. W. Edmonds, R. P. Campion, A. W. Rushforth, C. T. Foxon, and B. L. Gallagher, Appl. Phys. Lett. 103, 022401 (2013).

[31] H. X. Tang, R. K. Kawakami, D. D. Awschalom, and M. L. Roukes, Phys. Rev. B 74, 041310(R) (2006).

[32] J. Wunderlich, A. C. Irvine, J. Zemen, V. Holý, A. W. Rushforth, E. De Ranieri, U. Rana, K. Výborný, J. Sinova, C. T. Foxon, R. P. Campion, D. A. Williams, B. L. Gallagher, and T. Jungwirth, Phys. Rev. B 76, 054424 (2007).

[33] M. Yamanouchi, D. Chiba, F. Matsukura, and H. Ohno, Nature (London) 428, 539 (2004).

[34] M. Yamanouchi, D. Chiba, F. Matsukura, T. Dietl, and H. Ohno, Phys. Rev. Lett. 96, 096601 (2006).

[35] J.-P. Adam, N. Vernier, J. Ferré, A. Thiaville, V. Jeudy, A. Lemaitre, L. Thevenard, and G. Faini, Phys. Rev. B 80, 193204 (2009).

[36] J. Curiale, A. Lemaître, C. Ulysse, G. Faini, and V. Jeudy, Phys. Rev. Lett. 108, 076604 (2012).

[37] E. De Ranieri, P. E. Roy, D. Fang, E. K. Vehsthedt, A. C. Irvine, D. Heiss, A. Casiraghi, R. P. Campion, B. L. Gallagher, T. Jungwirth, and J. Wunderlich, Nat. Mater. 12, 808 (2013).

[38] L. Thevenard, A. Miard, L. Vila, G. Faini, A. Lemaître, N. Vernier, J. Ferré, and S. Fusil, Appl. Phys. Lett. 91, 142511 (2007).

[39] L. Thevenard, S. A. Hussain, H. J. von Bardeleben, M. Bernard, A. Lemaître, and C. Gourdon, Phys. Rev. B 85, 064419 (2012).

[40] Note, however, that various relative orientations of magnetic easy axis and spin-orbit field have been explored in metallic monodomain samples [70,71].

[41] All configurations $\pi$ rotated around the $z$ axis from those shown in Fig. 1 evidenced an identical behavior. We ruled out grounding issues, the effect of the LED illumination or transient fields emitted concurrently with the current pulses. An identical phenomenology was moreover observed on a $\mathrm{C}_{/ /}$device made out of a slightly more biaxial track, patterned by ion-beam etching this time.

[42] A. Vansteenkiste, J. Leliaert, M. Dvornik, M. Helsen, F. GarciaSanchez, and B. Van Waeyenberge, AIP Adv. 4, 107133 (2014).

[43] H. Kurebayashi, J. Sinova, D. Fang, A. C. Irvine, T. D. Skinner, J. Wunderlich, V. Novák, R. P. Campion, B. L. Gallagher, E. K. Vehstedt, L. P. Zârbo, K. Výborný, A. J. Ferguson, and T. Jungwirth, Nat. Nanotechnol. 9, 211 (2014).
[44] K. Obata and G. Tatara, Phys. Rev. B 77, 214429 (2008).

[45] A. Manchon and S. Zhang, Phys. Rev. B 78, 212405 (2008).

[46] X. Wang and A. Manchon, Phys. Rev. Lett. 108, 117201 (2012).

[47] K.-W. Kim, H.-W. Lee, K. -J. Lee, and M. D. Stiles, Phys. Rev. Lett. 111, 216601 (2013).

[48] The term fieldlike has somewhat confusingly been used to describe the spin-relaxation transfer torque $\left(\beta_{s r}\right.$ term) whose effect is absolutely transparent to the domains' charge/polarity. Here we use this term in its literal sense.

[49] E. Martinez, S. Emori, and G. S. D. Beach, Appl. Phys. Lett. 103, 072406 (2013).

[50] For out-of-plane metallic structures, Néel DWs may be favored over Bloch ones because of the spin-orbit interaction-induced Dzyaloshinkii-Moriya interaction [72]. In ( $\mathrm{Ga}, \mathrm{Mn}) \mathrm{As}$, there is also very likely a weak DMI: an interfacial term linked to the symmetry breaking at the interface [73], and a bulk one stemming from the Dresselhaus spin-orbit contribution [74]. Both will give a small Bloch component to the DW, but which will have to compete with the strong in-plane anisotropy [75].

[51] G. Yu, P. Upadhyaya, Y. Fan, J. G. Alzate, W. Jiang, K. L. Wong, S. Takei, S. A. Bender, L.-T. Chang, Y. Jiang, M. Lang, J. Tang, Y. Wang, Y. Tserkovnyak, P. K. Amiri, and K. L. Wang, Nat. Nanotechnol. 9, 1 (2014).

[52] A. K. Nguyen, R. V. Shchelushkin, and A. Brataas, Phys. Rev. Lett. 97, 136603 (2006).

[53] Since $P_{c}<0, \beta / \alpha$ is usually expressed positively. Here we add the minus sign to emphasize the sign of the mobility under current.

[54] K. W. Kim, K. J. Lee, H. W. Lee, and M. D. Stiles, Phys. Rev. B 92, 224426 (2015).

[55] M. Viret, D. Vignoles, D. Cole, J. M. D. Coey, W. Allen, D. S. Daniel, and J. F. Gregg, Phys. Rev. B 53, 8464 (1996).

[56] P. M. Levy and S. Zhang, Phys. Rev. Lett. 79, 5110 (1997).

[57] J. H. Franken, M. Hoeijmakers, H. J. M. Swagten, and B. Koopmans, Phys. Rev. Lett. 108, 037205 (2012).

[58] Z. Yuan and P. J. Kelly, Phys. Rev. B 93, 224415 (2016).

[59] G. Tatara and H. Kohno, Phys. Rev. Lett. 92, 086601 (2004).

[60] D. Matsubayashi, M. Udagawa, and M. Ogata, J. Phys. Soc. Jpn. 78, 033703 (2009).

[61] S. Bohlens and D. Pfannkuche, Phys. Rev. Lett. 105, 177201 (2010).

[62] K. M. D. Hals, A. K. Nguyen, and A. Brataas, Phys. Rev. Lett. 102, 256601 (2009).

[63] M. Hayashi, L. Thomas, C. Rettner, R. Moriya, Y. B. Bazaliy, and S. S. P. Parkin, Phys. Rev. Lett. 98, 037204 (2007).

[64] M. Dyakonov and V. Perel, Phys. Lett. A 35, 459 (1971).

[65] A. Manchon and K.-J. Lee, Appl. Phys. Lett. 99, 022504 (2011).

[66] A. Bisig, C. A. Akosa, J.-H. Moon, J. Rhensius, C. Moutafis, A. von Bieren, J. Heidler, G. Kiliani, M. Kammerer, M. Curcic, M. Weigand, T. Tyliszczak, B. Van Waeyenberge, H. Stoll, G. Schütz, K.-J. Lee, A. Manchon, and M. Kläui, Phys. Rev. Lett. 117, 277203 (2016).

[67] C.-Y. You, I. M. Sung, and B.-K. Joe, Appl. Phys. Lett. 89, 222513 (2006).

[68] J. S. Blakemore, J. Appl. Phys. 53, R123 (1982). 
[69] P. P. J. Haazen, E. Murè, J. H. Franken, R. Lavrijsen, H. J. M. Swagten, and B. Koopmans, Nat. Mater. 12, 299 (2013).

[70] L. Liu, C.-F. Pai, Y. Li, H. W. Tseng, D. C. Ralph, and R. A. Buhrman, Science (New York, N.Y.) 336, 555 (2012).

[71] S. Fukami, T. Anekawa, C. Zhang, and H. Ohno, Nat. Nanotechnol. 11, 621 (2016).
[72] A. N. Bogdanov and U. K. Rößler, Phys. Rev. Lett. 87, 037203 (2001).

[73] H. Imamura, P. Bruno, and Y. Utsumi, Phys. Rev. B 69, 121303(R) (2004).

[74] D. F. Mross and H. Johannesson, Phys. Rev. B 80, 155302 (2009).

[75] V. P. Kravchuk, J. Magn. Magn. Mater. 367, 9 (2014). 